\title{
Correspondence
}

\section{Single-RF Spatial Modulation Requires Single-Carrier \\ 2 Transmission: Frequency-Domain Turbo \\ 3 Equalization for Dispersive Channels}

4

5

\author{
Shinya Sugiura, Senior Member, IEEE, and \\ Lajos Hanzo, Fellow, IEEE
}

6 Abstract-In this paper, we propose a broadband single-carrier (SC) 7 spatial modulation (SM)-based multiple-input-multiple-output (MIMO) 8 architecture relying on a soft decision (SoD) frequency-domain equal9 ization (FDE) receiver. We demonstrate that conventional orthogonal-

10 frequency-division-multiplexing (OFDM)-based broadband transmissions

11 are not readily suitable for the single-radio-frequency-assisted SM-MIMO

12 schemes since this scheme exhibits no substantial performance advantage

13 over single-antenna transmissions. To circumvent this limitation, a low-

14 complexity SoD FDE algorithm based on the minimum mean square error

15 (MMSE) criterion is invoked for our broadband SC-based SM-MIMO

16 scheme, which is capable of operating in a strongly dispersive channel

17 having a long channel impulse response at moderate decoding complexity.

18 Furthermore, our SoD FDE attains a near-capacity performance with the

19 aid of a three-stage concatenated SC-based SM architecture.

20 Index Terms-Author, please supply index terms/keywords for your 22 paper. To download the IEEE Taxonomy go to http://www.ieee.org/ documents/taxonomy_v101.pdf.

\section{INTRODUCTION}

24 Spatial modulation (SM)-based multiple-input-multiple-output 25 (MIMO) designs have become popular as a benefit of their low-cost 26 single radio frequency (RF) transmitters and their ability to increase 27 the attainable transmission rates [1]-[3]. The information bits of the $28 \mathrm{SM}$ transmitter are mapped to both the spatial (antenna) dimension 29 and to the classic amplitude phase-shift keying (APSK) constellation. 30 More specifically, one of the $M$ transmit antenna (TA) elements 31 is activated by $\log _{2} M$ information bits, whereas a complex-valued 32 APSK symbol $s_{l}$, which is constituted by $\log _{2} \mathcal{L}$ information bits, is 33 transmitted from the activated TA. Hence, a total of $B=\log _{2}(\mathcal{L} \cdot M)$ 34 bits are conveyed during each symbol interval by using a single-RF35 based transmitter.

36 Current wireless telecommunication standards typically employ 37 broadband techniques [4], such as orthogonal frequency-division mul38 tiplexing (OFDM) [5] and single-carrier (SC) frequency-division mul39 tiple access [6]. However, the majority of previous SM studies has 40 focused on narrowband scenarios, assuming that the SM symbols are 41 transmitted over a frequency-flat channel [7]-[11].

42 Nevertheless, some OFDM-based broadband SM schemes have 43 also been developed [12], [13]; these are, however, less attractive

Manuscript received June 24, 2014; revised October 2, 2014; accepted November 11, 2014. This work was supported by the Japan Society for the Promotion of Science Grants-in Aid for Scientific Research (KAKENHI) under Grant 26709028. The review of this paper was coordinated by Dr. C. Cozzo.

S. Sugiura is with the Department of Computer and Information Sciences, Tokyo University of Agriculture and Technology, Tokyo 184-8588, Japan (e-mail: sugiura@ieee.org).

L. Hanzo is with the School of Electronics and Computer Science,University of Southampton, Southampton SO17 1BJ, U.K. (e-mail: 1h@ecs.soton.ac.uk).

Color versions of one or more of the figures in this paper are available online at http://ieeexplore.iee.org.

Digital Object Identifier 10.1109/TVT.2014.2370679 from a practical point of view, although this has not been explicitly 44 detailed before. For instance, let us assume that the SM scheme's TA 45 activation process is individually implemented for each subcarrier of 46 an OFDM system. This requires that multiple TA elements have to be 47 simultaneously activated over the OFDM frame, hence precluding the 48 benefit of having the abovementioned single-RF-based SM scheme. 49 In practice, to maintain a single-RF SM transmitter structure, the 50 previously proposed OFDM-based SM schemes [12], [13] have to 51 rely on block-based antenna activation, in which the TA activation 52 process is carried out for each OFDM frame, rather than for each 53 subcarrier. ${ }^{1}$ In this architecture, the SM scheme's contribution to the 54 rate increase per subcarrier becomes as low as $\left(\log _{2} M\right) / N_{C}$, where 55 $N_{C}$ is the number of subcarriers. This gain is $N_{C}$ times lower than 56 that expected in a narrowband SM-MIMO scenario. In this sense, the 57 OFDM-based SM scheme's advantage over the conventional single- 58 antenna-aided system is negligible for a practical broadband scenario, 59 in which hundreds of subcarriers are supported. In general, the same 60 holds not only for the SM scheme but also for most of the MIMO 61 schemes relying on a single-RF transmitter [15]-[17]. However, this 62 issue has not been explicitly considered, in spite of its significant 63 importance in terms of realistic broadband communications. ${ }^{2} \quad 64$

The broadband SC-based SM architecture has the potential of 65 solving the problems of the abovementioned OFDM-based SM-MIMO 66 schemes. Since the SM scheme's TA activation process is carried out 67 for each symbol in the SC-based SM architecture, the benefits of an 68 increased transmission rate and a low-cost single-RF transmitter are 69 maintained, while facilitating its operation as a broadband system. 70 So far, only very few SC-based SM schemes capable of operating 71 in dispersive channels have been developed [18]-[20]. In [18], the 72 SM scheme's TA activation concept was combined with frequency- 73 shift keying modulation, which spreads the transmitted signal not 74 only across the spatial domain but across the frequency domain (FD) 75 as well. In [19], a cyclic prefix (CP)-based SC-MIMO scheme was 76 developed, which relied on exhaustive maximum likelihood (ML) 77 detection. In [20], zero padding (ZP)-aided SC-SM schemes based on 78 time domain (TD) ML equalization and reduced-complexity parallel- 79 interference cancelation were proposed to achieve the maximum at- 80 tainable transmit and receive diversity gains. However, the frame 81 length and the channel impulse response (CIR) length considered in 82 [18]-[20] was less than ten taps, although the CIR length of practical 83 broadband channels is often significantly higher. More importantly, 84 all the previous SC-SM schemes [18]-[20] were developed for hard- 85 decision-based receivers, which prevents us from exploiting the bene- 86 fits of powerful iterative detection.

To eliminate the effects of long CIRs encountered in practical 88 broadband dispersive channels, an efficient equalization algorithm has 89 to be conceived for the SC-SM scheme. Furthermore, to employ a 90

\footnotetext{
${ }^{1}$ When considering a full-RF SM-MIMO transmitter that is equipped with the same number of RF chains as that of the TA elements, as shown in [14], the subcarrier-based OFDM-SM system is capable of operating without imposing a penalty on the transmission rate. However, such a full-RF transmitter imposes a higher terminal cost than its single-RF counterpart.

${ }^{2}$ To provide further insights, studies of conventional single-RF MIMO schemes have focused, for simplicity, on narrowband scenarios associated with frequency-flat fading. However, unlike for its full-RF MIMO counterparts, its application to broadband transmissions is not straightforward. This challenge is tackled in this paper.
} 
TABLE I

Fundamental COMPARISONS BETWEen THE BROADBAND SM-MIMO SCHEMES

\begin{tabular}{|c|c|c|c|}
\hline$\overline{\overline{\text { Type }}}$ & Normalized transmission rate $[\mathrm{bps} / \mathrm{Hz}]$ & Number of RF chains & Number of transmit antennas \\
\hline Proposed SC-based SM-MIMO & $\log _{2}(M \cdot \mathcal{L})$ & 1 & $M$ \\
\hline Subcarrier-based OFDM-SM-MIMO & $\log _{2}(M \cdot \mathcal{L})$ & $M$ & $M$ \\
\hline 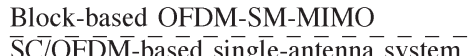 & $\log _{2}(\mathcal{L})+\left(\log _{2} M\right) / N_{C}$ & -1 & $\frac{M}{1}$ \\
\hline SC/OFDM-based single-antenna system & & & \\
\hline
\end{tabular}

91 powerful channel-coding scheme relying on iterative detection, the 92 SC-SM detector has to output soft information. In the context of classic 93 single-antenna-based or MIMO arrangements, an efficient soft deci94 sion (SoD) frequency-domain equalization (FDE) was proposed and 95 standardized for the Long-Term Evolution system [21]. To the best of 96 our knowledge, an efficient SoD equalization algorithm that is capable 97 of exchanging extrinsic information with a powerful channel-coding 98 scheme relying on soft-input soft-output (SISO) iterative detection has 99 not been conceived for a broadband SC-based SM scheme. ${ }^{3}$

100 Against this background, the novel contributions of this paper are as 101 follows.

103

104

105

106

107

108

109

110

111

112

113

114

115

116

117

118

119

120

121

122 The remainder of this paper is organized as follows. In Section II, we 123 describe the model of our broadband SC-based SM scheme, whereas in 124 Section III, we present our FDE algorithm. In Section IV, the proposed 125 scheme's iterative convergence behavior and maximum achievable 126 limit are analyzed. In Section V, we consider the performance of our 127 system, whereas our conclusions are presented in Section VI.

\section{SYSTEM MODEL}

129 Here, we commence by clarifying our motivation of designing an 130 SC-based SM-MIMO system, rather than its OFDM-based counter131 part. Then, we outline the model of our SC-based SM-MIMO system.

\section{A. Preliminary Discussions of Our Broadband 133 SM-MIMO Scheme}

134 Before detailing the proposed SC-based SM-MIMO system, we 135 introduce the broadband SM-MIMO family and analyze the limitations 136 imposed on the previous OFDM-based SM-MIMO system.

\footnotetext{
${ }^{3}$ Assuming single-RF SM-MIMO transmissions, the SM-specific shaping filter has to be designed so that the pulse is isolated in the TD. This may reduce the bandwidth efficiency and the power amplifier efficiency in comparison with a classic modem employing an efficient raised-cosine filter. However, this issue is beyond the scope of this paper; the details are discussed in [22].
}

The bandwidth efficiency of a conventional OFDM- or SC-based 137 single-antenna system is given by

$$
R_{\text {SISO }}=\log _{2} \mathcal{L}[\mathrm{bps} / \mathrm{Hz}]
$$

where $\mathcal{L}$ is the constellation size. For simplicity, we assume that the 139 relative overhead of the guard interval or CP over the frame length is 140 sufficiently low.

Next, let us consider the OFDM-based SM-MIMO scheme relying 142 on a single-RF transmitter. As briefly mentioned in the introduction, 143 a single-RF transmitter is unable to simultaneously activate multiple 144 TA elements. Hence, the entire OFDM frame, including the $N_{\mathrm{C}} 145$ $\mathcal{L}$-PSK/quadrature amplitude modulation (QAM)-based subcarriers, 146 must be transmitted by a single activated TA element. The bandwidth 147 efficiency of the OFDM-based SM-MIMO system is

$$
R_{\mathrm{SM}-\mathrm{MIMO}}^{\text {(OFD) }}=\log _{2} \mathcal{L}+\frac{\log _{2} M}{N_{\mathrm{C}}}[\mathrm{bps} / \mathrm{Hz}]
$$

where the first term represents the classic $\mathcal{L}$-point APSK-modulated 149 subcarrier, whereas the second term corresponds to the TA activation 150 process carried out per OFDM frame. Note that $R_{\text {SM-MIMO }}^{\text {(OFDM }}$ in (2) 151 increases to $\log _{2}(M \cdot \mathcal{L})$ in the full-RF SM-MIMO transmitter, since 152 the SM scheme's antenna activation process is carried out for each 153 subcarrier. However, this is achieved at the price of increasing the 154 transmitter's cost, hence relinquishing the main benefit of the single- 155 RF SM scheme.

By contrast, the SC-based SM-MIMO architecture facilitates the 157 SM scheme's independent TA activation process for each symbol, i.e., 158 one of the $M$ TA elements is activated during each symbol interval, 159 and the $\mathcal{L}$-size PSK/QAM symbol is transmitted from the activated 160 TA. Therefore, the bandwidth efficiency of the SC-based SM-MIMO 161 system becomes 162

$$
R_{\mathrm{SM}-\mathrm{MIMO}}^{\mathrm{SC}}=\log _{2} \mathcal{L}+\log _{2} M[\mathrm{bps} / \mathrm{Hz}]
$$

which reflects the expected throughput gain of the SM scheme [2], 163 [3]. However, note again that this is not attainable by the OFDM- 164 based single-RF SM architecture represented by (2). The fundamental 165 comparisons between the various broadband SM-MIMO schemes are 166 shown in Table I and Fig. 1.

\section{B. Model of Our SC-Based SM-MIMO Scheme}

Let us consider a broadband SC-SM transmitter having $M$ TAs 169 and using an $\mathcal{L}$-sized PSK/QAM modulation scheme. Similar to the 170 narrowband SM scheme, each SM symbol contains $B_{1}=\log _{2} M 171$ and $B_{2}=\log _{2} \mathcal{L}$ information bits, where one of the $M$ TAs is acti- 172 vated according to $B_{1}$ bits, whereas the $B_{2}$ bits are mapped onto a 173 PSK/QAM symbol $s_{l}(k)$. Furthermore, $k$ is the symbol index, and we 174 denote the index of the activated TA during the $k$ th interval by $m(k) .175$ For simplicity, we employ a vectorial notation for the SM symbol, as 176 follows:

$$
\mathbf{s}(k)=[\underbrace{0, \ldots, 0}_{m(k)-1}, s_{l}(k), \underbrace{0, \ldots, 0}_{M-m(k)}]^{T} \in \mathbb{C}^{M \times 1} .
$$




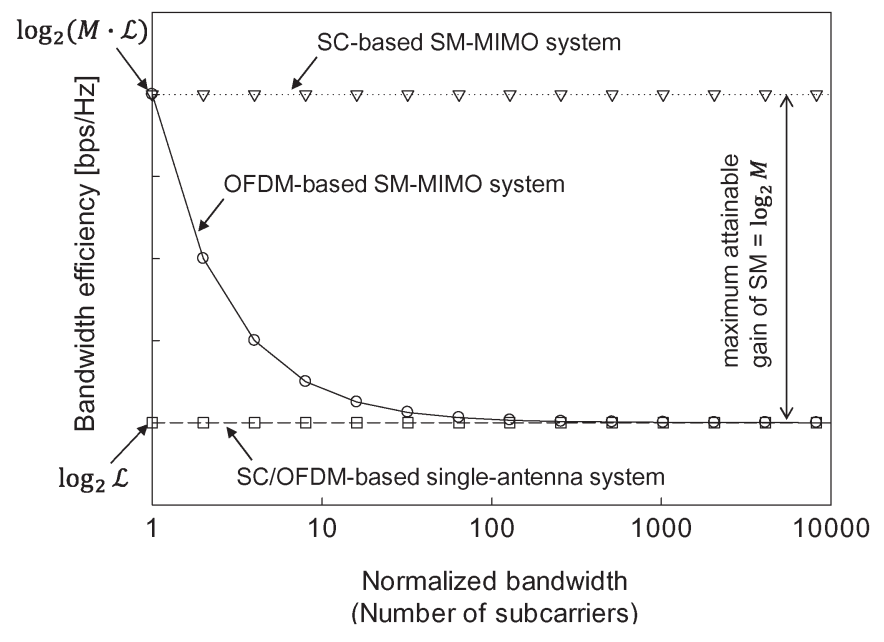

Fig. 1. Bandwidth efficiencies of the broadband OFDM- and SC-based SM-MIMO systems; that of the classic SC/OFDM-based single-antenna system is included as a benchmark.

178 We consider block transmissions of the $K$ SM symbols, i.e.,

$$
\mathbf{S}=[\mathbf{s}(1), \ldots, \mathbf{s}(K)]^{T} \in \mathbb{C}^{M \times K} .
$$

179 After concatenating the $\nu$-length $\mathrm{CP}$, which is higher than the CIR 180 length $\xi$, the SM symbol block is transmitted over $(K+\nu)$ consecutive 181 symbol durations.

182 At the receiver, the $\nu$-length $\mathrm{CP}$ is removed from the received $(K+$ $183 \nu$ )-length SM block. Then, we arrive at

$$
\begin{aligned}
\mathbf{y}= & {\left[y_{1}(1), \ldots, y_{1}(K), \ldots\right.} \\
& \left.y_{N}(1), \ldots, y_{N}(K)\right]^{T} \in \mathbb{C}^{N K \times 1} \\
= & \mathbf{H} \overline{\mathbf{s}}+\mathbf{n}
\end{aligned}
$$

184 where $\overline{\mathbf{s}} \in \mathbb{C}^{M K \times 1}$ is given by a vector stacking operation applied to $185 \mathrm{~S}$. Furthermore, $N$ is the number of receive antenna elements, whereas $186 \mathbf{n} \in \mathbb{C}^{N \times 1}$ denotes the associated additive noise components, where 187 the random variables are distributed according to the complex-valued 188 Gaussian distribution $\mathcal{C N}\left(0, N_{0}\right)$, with zero mean and variance $N_{0}$. 189 Moreover, the channel components $\mathbf{H} \in \mathbb{C}^{N K \times M K}$ are expressed as 190 submatrices, as follows:

$$
\mathbf{H}=\left[\begin{array}{ccc}
\mathbf{H}_{11} & \cdots & \mathbf{H}_{1 M} \\
\vdots & \ddots & \vdots \\
\mathbf{H}_{N 1} & \cdots & \mathbf{H}_{N M}
\end{array}\right]
$$

191 where each submatrix $\mathbf{H}_{n m} \in \mathbb{C}^{K \times K}$ represents a circular matrix, 192 which is composed of the $\xi$-length CIRs $\mathbf{h}_{n m}=\left[h_{n m}^{(1)}, \ldots, h_{n m}^{(\xi)}\right]^{T} \in$ $193 \mathbb{C}^{\xi \times 1}$, while assuming the relationship of $\xi \leq \nu<K$.

\section{III. FREQUENCY DOMAIN EQUALIZATION-AIDED \\ 195 SINGLE-CARRIER-SPATIAL MOdULATION 196 Multiple-InPut-Multiple-OutPut ReCEIVER}

197 Here, we derive our hard-decision SC-SM FDE receiver and then 198 extend it to its SoD counterpart, which is suitable for iterative detection 199 and is based on the turbo principle [23].

\section{A. Hard-Decision SC-SM Receiver}

201 With the aid of fast Fourier transforms (FFTs), each channel subma202 trix $\mathbf{H}_{n m}$ is represented by

$$
\mathbf{H}_{n m}=\mathbf{Q}^{T} \boldsymbol{\Lambda}_{n m} \mathbf{Q}^{*}
$$

where the element in the $k$ th row and $l$ th column of $\mathbf{Q}$ is 203 given by $[\mathbf{Q}]_{k l}=(1 / \sqrt{K}) \exp [-2 \pi j(k-1)(l-1) / K]$. Further- 204 more, $\Lambda_{n m} \in \mathbb{C}^{K \times K}$ denotes the diagonal matrix for which the 205 nonzero elements are the $K$ FFT coefficients. Hence, the received 206 signals of (7) can be rewritten as

$$
\begin{aligned}
\mathbf{y} & =\left(\mathbf{I}_{N} \otimes \mathbf{Q}^{T}\right) \mathbf{\Lambda}\left(\mathbf{I}_{\mathbf{M}} \otimes \mathbf{Q}^{*}\right) \overline{\mathbf{s}}+\mathbf{n} \\
& =\left(\mathbf{I}_{N} \otimes \mathbf{Q}^{T}\right) \mathbf{\Lambda} \mathbf{s}_{f}+\mathbf{n}
\end{aligned}
$$

where we have

$$
\begin{aligned}
\mathbf{\Lambda} & =\left[\begin{array}{ccc}
\boldsymbol{\Lambda}_{11} & \cdots & \boldsymbol{\Lambda}_{1 M} \\
\vdots & \ddots & \vdots \\
\boldsymbol{\Lambda}_{N 1} & \cdots & \boldsymbol{\Lambda}_{N M}
\end{array}\right] \in \mathbb{C}^{N K \times M K} \\
\mathbf{s}_{f} & =\left(\mathbf{I}_{\mathbf{M}} \otimes \mathbf{Q}^{*}\right) \overline{\mathbf{s}} \in \mathbb{C}^{M K \times 1} .
\end{aligned}
$$

Moreover, $\mathbf{I}_{n} \in \mathbb{R}^{n \times n}$ is the $n$-size identity matrix, and $\otimes$ represents 209 the Kronecker product.

Upon multiplying both sides of (11) by $\left(\mathbf{I}_{N} \otimes \mathbf{Q}^{*}\right)$, we arrive at the 211 received signals $\mathbf{y}_{f}$ in the $\mathrm{FD}$, as follows:

$$
\mathbf{y}_{f}=\Lambda \mathbf{s}_{f}+\mathbf{n}_{f}
$$

where $\mathbf{n}_{f}=\left(\mathbf{I}_{N} \otimes \mathbf{Q}^{*}\right) \mathbf{n}$. Next, MMSE filtering is invoked for es- 213 timating the FD SC-SM signals $\mathbf{s}_{f}$ by minimizing the average MSE 214 between the FD SM symbols $\mathbf{s}_{f}$ and the estimates $\hat{\mathbf{s}}_{f}$. Given the 215 complex-valued weights $\mathbf{w} \in \mathbb{C}^{N K \times 1}$, the MMSE-filtered outputs are 216 given by

$$
\hat{\mathbf{s}}_{f}=\mathbf{w}^{T} \mathbf{y}_{f} .
$$

According to [25], the complex-valued MMSE equalizer weights w 218 are calculated as follows:

$$
\begin{aligned}
\mathbf{w} & =\left(\mathbf{R}_{y y}\right)^{-1} \mathbf{R}_{y s} \\
& =\left(\frac{\boldsymbol{\Lambda} \boldsymbol{\Lambda}^{H}}{M}+N_{0} \mathbf{I}_{N K}\right)^{-1} \frac{\boldsymbol{\Lambda}}{M}
\end{aligned}
$$

where we have

$$
\begin{aligned}
& \mathbf{R}_{y y}=\mathbb{E}\left[\mathbf{y}_{f} \mathbf{y}_{f}^{H}\right]=\frac{\boldsymbol{\Lambda} \boldsymbol{\Lambda}^{H}}{M}+N_{0} \mathbf{I}_{N K} \\
& \mathbf{R}_{y s}=\mathbb{E}\left[\mathbf{y}_{f} \mathbf{s}_{f}^{H}\right]=\frac{\boldsymbol{\Lambda}}{M}
\end{aligned}
$$

while

$$
\begin{aligned}
\mathbb{E}\left[\mathbf{s}_{f} \mathbf{s}_{f}^{H}\right] & =\mathbb{E}\left[\left(\mathbf{I}_{\mathbf{M}} \otimes \mathbf{Q}^{*}\right) \overline{\mathbf{s}} \overline{\mathbf{s}}^{H}\left(\mathbf{I}_{\mathbf{M}} \otimes \mathbf{Q}^{T}\right)\right] \\
& =\frac{\mathbf{I}_{M K}}{M} .
\end{aligned}
$$

Note that in the terms that include the coefficient $M, \mathbf{R}_{y y}$, and $\mathbf{R}_{y s} 222$ of (18) and (19) are different from those derived for conventional 223 equalization or for the traditional MIMO systems. This is because the 224 SM symbol $\mathbf{s}(k)$ contains only a single nonzero element and because 225 the sparsity factor of $\overline{\mathbf{s}}$ is $M$, as shown in (20).

Next, we convert the FD estimates $\hat{\mathbf{s}}_{f}$ of (15) into their TD counter- 227 parts, as follows:

$$
\hat{\overline{\mathbf{s}}}=\left(\mathbf{I}_{M} \otimes \mathbf{Q}^{T}\right) \hat{\mathbf{s}}_{f} .
$$

By rearranging the vector $\hat{\overline{\mathbf{s}}}$, we arrive at the SC-SM estimates of

$$
\hat{\mathbf{S}}=[\hat{\mathbf{s}}(1), \ldots, \hat{\mathbf{s}}(K)]^{T}
$$

which corresponds to the transmitted SM frame $\mathbf{S}$ shown in (5). 


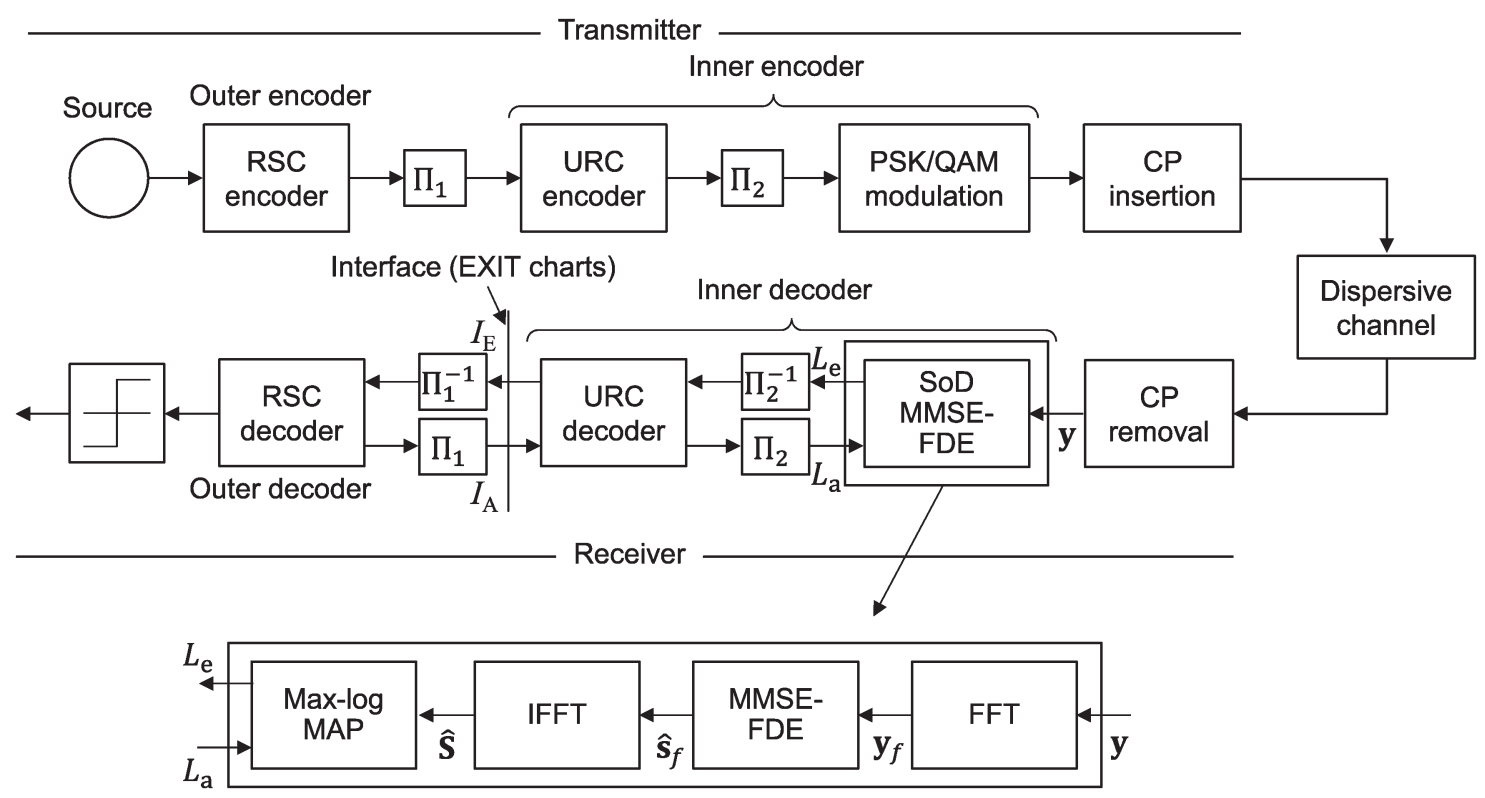

Fig. 2. Transceiver architecture of our broadband SC-based SM scheme.

231 Finally, symbol-based ML detection is applied to $\hat{\mathbf{S}}$

$$
\langle\hat{m}(k), \hat{l}(k)\rangle=\arg \min _{m, l}\left\|\hat{\mathbf{s}}(k)-\mathbf{s}_{m, l}\right\|^{2}
$$

232 where we have

$$
\mathbf{s}_{m, l}=[\underbrace{0, \ldots, 0}_{m-1}, s_{l}, \underbrace{0, \ldots, 0}_{M-m}]^{T} \in \mathbb{C}^{M \times 1} .
$$

233 Note that (23) represents symbol-by-symbol ML detection, which is 234 equivalent to additive white Gaussian noise, and hence, it is indepen235 dent both of the CIR length $\xi$ as well as of the frame length $K$. This 236 allows us to benefit from the SM scheme's low decoding complexity.

\section{B. SoD SC-SM Receiver}

238 Here, we extend the hard-decision SC-SM receiver derived in the 239 previous section to its SoD version. Typically, the MMSE-based SoD 240 MIMO receiver employs the soft-interference cancelation concept 241 proposed in [26]. However, in our SC-SM scheme, it is a challenging 242 task to compute soft SM symbols from the a priori information, due to 243 the SM-specific TA activation principle. ${ }^{4}$

244 Instead of the hard-decision ML detection of (23), we simply carry 245 out SoD maximum a posteriori (MAP) demodulation. By using the 246 intersymbol-interference-free estimates of the SM symbol vector $\hat{\mathbf{s}}(k)$ 247 shown in (22), we arrive at the extrinsic log-likelihood ratio (LLR) 248 value of the bit $b_{p}\left(p=1, \ldots, \log _{2}(M \cdot \mathcal{L})\right)$, which is included in the $249 k$ th SM symbol, as follows [9]:

$$
\begin{aligned}
L_{\mathrm{e}}\left(b_{p}\right)= & \max _{\mathbf{s}_{m, l} \in \mathbf{s}_{1}^{p}}\left[-\frac{\left\|\hat{\mathbf{s}}(k)-\mathbf{s}_{m, l}\right\|}{N_{\mathrm{MAP}}}+\sum_{j \neq k} b_{j} L_{\mathrm{a}}\left(b_{j}\right)\right] \\
& -\max _{\mathbf{s}_{m, l} \in \mathbf{s}_{0}^{p}}\left[-\frac{\left\|\hat{\mathbf{s}}(k)-\mathbf{s}_{m, l}\right\|}{N_{\mathrm{MAP}}}+\sum_{j \neq k} b_{j} L_{\mathrm{a}}\left(b_{j}\right)\right]
\end{aligned}
$$

\footnotetext{
${ }^{4}$ To expound further, since the SM mapping scheme attained by antenna activation is discrete, it is difficult to define the soft values.
}

where $\mathbf{s}_{1}^{p}$ and $\mathbf{s}_{0}^{p}$ represent the subspace of the legitimate equiva- 250 lent signals, satisfying $\mathbf{s}_{1}^{p} \equiv\left\{\mathbf{s}_{m, l} \in \mathbf{s}: b_{p}=1\right\}$ and $\mathbf{s}_{0}^{p} \equiv\left\{\mathbf{s}_{m, l} \in 251\right.$ $\left.\mathbf{s}: b_{p}=0\right\}$, respectively. Furthermore, $L_{\mathrm{a}}(\cdot)$ represents the a priori 252 information expressed in terms of LLRs, whereas $N_{\text {MAP }}$ denotes the 253 variance of the noise that was included in the SM symbol estimates 254 $\hat{\mathbf{s}}(k)$. Since the SoD demodulation of (25) is based on a symbol-by- 255 symbol operation similar to the hard-decision version of (23), low 256 complexity is maintained.

\section{EXIT-Chart-Aided SEMIANALYSis OF OUR FREQUENCY DOMAIN EQUALIZATION-AIDED Single-CARRIER-SPATIAL-MOdULATION SCHEME}

\section{A. Three-Stage Concatenated SC-SM Transceiver}

Fig. 2 shows our three-stage concatenated recursive systematic 263 convolutional (RSC)-coded and unity-rate convolutional (URC)-coded 264 SC-SM structures. The transmitter channel encodes the source infor- 265 mation bits using the RSC code, and these are then interleaved by the 266 first interleaver $\Pi_{1}$. The interleaved bits are then encoded by the URC 267 code, and these are then interleaved again by the interleaver $\Pi_{2}$. The 268 resultant bits are then mapped to the SC-SM symbols S. After adding 269 the $\mathrm{CP}$ symbols to $\mathbf{S}$, the $\mathrm{SM}$ symbols are transmitted.

As shown in Fig. 1, at the receiver, the CP symbols are removed 271 from the received signal block. Next, the SISO decoders (i.e., the SoD 272 FDE-aided SC-SM decoder proposed in Section III, the URC decoder, 273 and the RSC decoder) iteratively exchange their extrinsic information. 274 For each of the $I_{\text {out }}$ outer iterations, there are $I_{\text {in }}$ inner iterations carried 275 out between each SC-SM decoder and the associated URC decoder. 276 Therefore, the total number of iterations is $\left(I_{\text {in }} \cdot I_{\text {out }}\right)$. The details of 277 the three-stage concatenated system can be found in [27] and [28]. 278

\section{B. Convergence Behavior Analysis}

Here, we use EXIT charts [24] for visualizing the convergence 280 behavior of the iterative detection. We present the EXIT charts of our 281 SC-based SM scheme, where $M=N=4$ TAs and receive antennas 282 were used, whereas the signal-to-noise ratio (SNR) was varied from 283 0 to $10 \mathrm{~dB}$, in steps of $1 \mathrm{~dB}$. The outer code's EXIT curve is 284 also plotted for the half-rate $\operatorname{RSC}(2,1,2)$ code, having the octally 285 


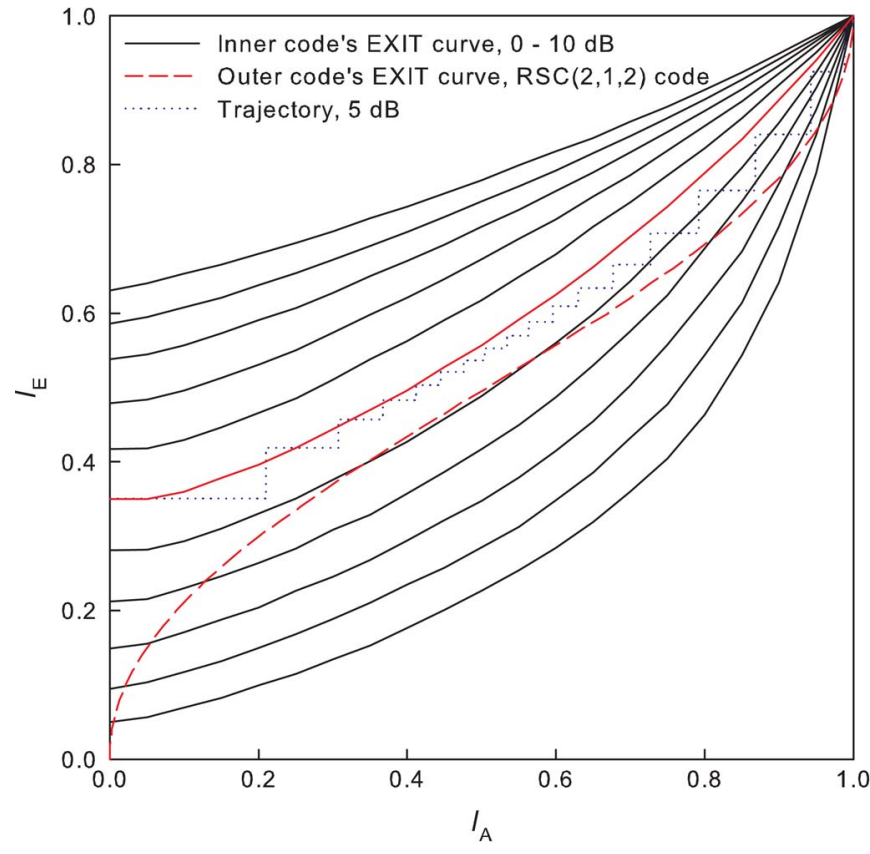

Fig. 3. EXIT charts of our MMSE-FDE-aided SC-based SM-MIMO system, employing $M=N=4$ transmit and receive antenna elements and 16-PSK modulation. The normalized transmission rate of the half-rate channel-encoded system was $R=3 \mathrm{bps} / \mathrm{Hz}$. The EXIT trajectory was calculated by assuming that the code length was 38400 bits and that the $\mathrm{SNR}=5 \mathrm{~dB}$.

286 represented generator polynomials of $\left(G_{\mathrm{r}}, G\right)=(3,2)$ [29], where $287 G_{\mathrm{r}}$ is the recursive feedback polynomial, and $G$ is the feedforward 288 polynomial. We assumed frequency-selective Rayleigh fading with 289 equal-power 15-length CIRs. Furthermore, an $(\mathcal{L}=16)$-PSK modu290 lation scheme was considered, and the normalized transmission rate 291 of the half-rate channel-encoded system was $R=3 \mathrm{bps} / \mathrm{Hz}$. The 292 EXIT trajectory was calculated by assuming that the code length was 29338400 bits, and we had SNR $=5 \mathrm{~dB}$. The number of inner iterations 294 was $I_{\text {in }}=2$. As seen in Fig. 3, upon increasing the SNR value, the 295 inner code's EXIT curve shifted upward, and an open tunnel emerged 296 between the inner code and outer code's EXIT curves at SNR = $2975 \mathrm{~dB}$, where the corresponding EXIT trajectory reached the perfect 298 convergence point of $\left(I_{\mathrm{A}}, I_{\mathrm{E}}\right)=(1,1)$ after $I_{\text {out }}=20$ outer iterations. 299 This ensured that an infinitesimally low bit error ratio (BER) was 300 achievable in the simulated SC-based $\mathrm{SM}$ scenario at $\mathrm{SNR}=5 \mathrm{~dB}$.

\section{C. Maximum Achievable Limit}

302 According to the area property of EXIT charts [30], the maximum 303 achievable limit of our FDE-based SC-SM scheme is calculated as 304 follows: $C_{\text {EXIT }}=\mathcal{A}(\rho) R_{\mathrm{SM}-\mathrm{MIMO}}^{\mathrm{(SC}}[\mathrm{bps} / \mathrm{Hz}]$, where $\mathcal{A}(\rho)$ represents the 305 area under the inner code's EXIT curve at $\mathrm{SNR}=\rho$. The benefit of 306 using this metric is that we have the potential of evaluating any SoD 307 detectors in a semianalytical manner, while it is typically a challenging 308 task to derive the theoretical limit of a suboptimal SoD detector. Since 309 the target scenario of our SC-based SM scheme has a long CIR, the 310 theoretical limit of the optimal detector is not attainable due to its 311 excessive calculation complexity.

312 Fig. 4 shows the maximum achievable rates of our SC-based SM 313 scheme, relying on $M=4$ TAs and $N=4$ receive antennas, while 314 the modulation schemes are considered to be quadrature phase-shift 315 keying, 8-PSK, and 16-PSK. The other system parameters are the same 316 as those used in Fig. 3. The associated capacity of the continuous317 input-continuous-output memoryless channel (CCMC) is also shown

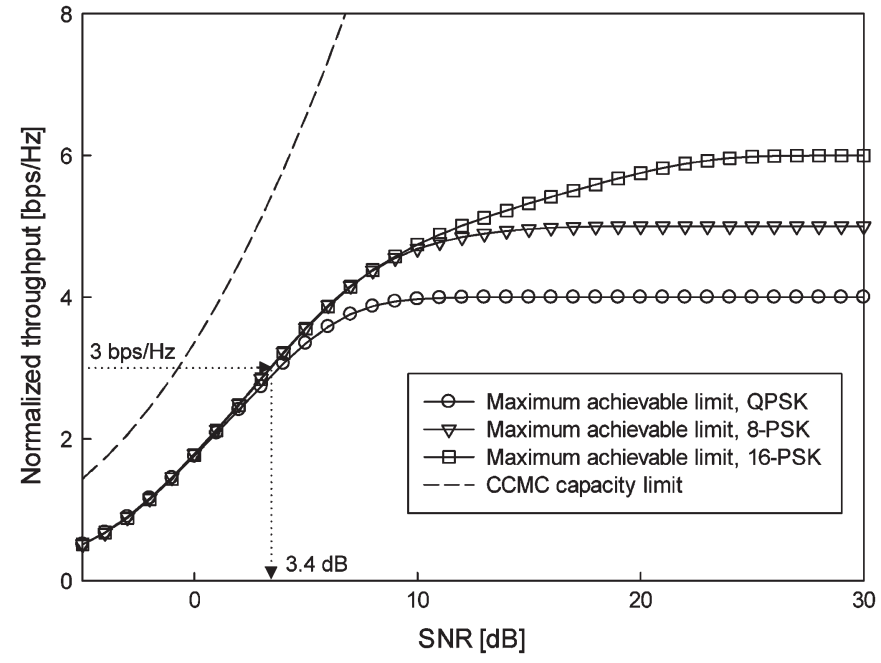

Fig. 4. Maximum achievable limits of our SC-SM-MIMO schemes, employing $M=N=4$ transmit and receive antenna elements and 4-, 8-, and 16-PSK modulation schemes. The associated CCMC capacity limit is also shown.

as a benchmark, representing unconstrained signaling. Observe in 318 Fig. 4 that upon increasing the constellation size from $\mathcal{L}=4$ to $\mathcal{L}=319$ 16 , the maximum achievable limit at high SNR is increased. In each 320 scenario, the curve reached the rate formulated in (3). When employing 321 the half-rate RSC code for the 16-PSK SC-based SM scheme, the 322 limit was reached for an SNR of $\rho=3.4 \mathrm{~dB}$. Since the code's EXIT 323 curve is based on the soft output of the inner code, the conven- 324 tional hard-decision SC-based SM detector is not applicable in this 325 evaluation.

\section{BER PERFORMANCE}

A. Channel-Encoded SC-SM Scheme Aided by Iterative Detection

Here, we investigate the BER of our SC-based SM scheme. The 331 basic system parameters used in our simulations were the same as 332 those in Fig. 3. For simplicity, the estimate of the noise variance 333 $N_{\text {MAP }}$ shown in (4) was set to $N_{0}$. We considered a frequency-selective 334 Rayleigh distributed block-fading channel, where the block length was 335 $K=256$, the CP length was $\nu=32$, and the CIR taps were constant 336 for a block, but were independently faded for the consecutive blocks. ${ }^{5} 337$

Fig. 5 shows the achievable BER of our FDE-aided SC-SM scheme, 338 where the basic system parameters were the same as those used in 339 the EXIT charts in Fig. 3. The number of outer iterations $I_{\text {out }}$ was 340 varied from 0 to 16. Observe in Fig. 5 that upon increasing the number 341 of outer iterations $I_{\text {out }}$, the BER curve significantly improved. In 342 particular, an infinitesimally low BER was achieved for SNR $=5 \mathrm{~dB} 343$ with the aid of $I_{\text {out }}=16$ outer iterations, as predicted by the associated 344 EXIT charts shown in Fig. 3. This is the explicit benefit of the proposed 345 turbo FDE scheme's iterative detection, which would not be attainable 346 by the previous hard-decision SC-SM schemes [18]-[20].

\section{Conclusion}

In conclusion, single-RF SM requires SC transmissions, rather 349 than OFDM, for transmission over practical broadband SM-MIMO 350

\footnotetext{
${ }^{5}$ Note that the power penalty per frame imposed by the $\mathrm{CP}$ overhead was as low as $-10 \log _{10}[K /(K+\nu)]=0.51 \mathrm{~dB}$. This can be further reduced by increasing the block length, at the cost of increasing the delay.
} 


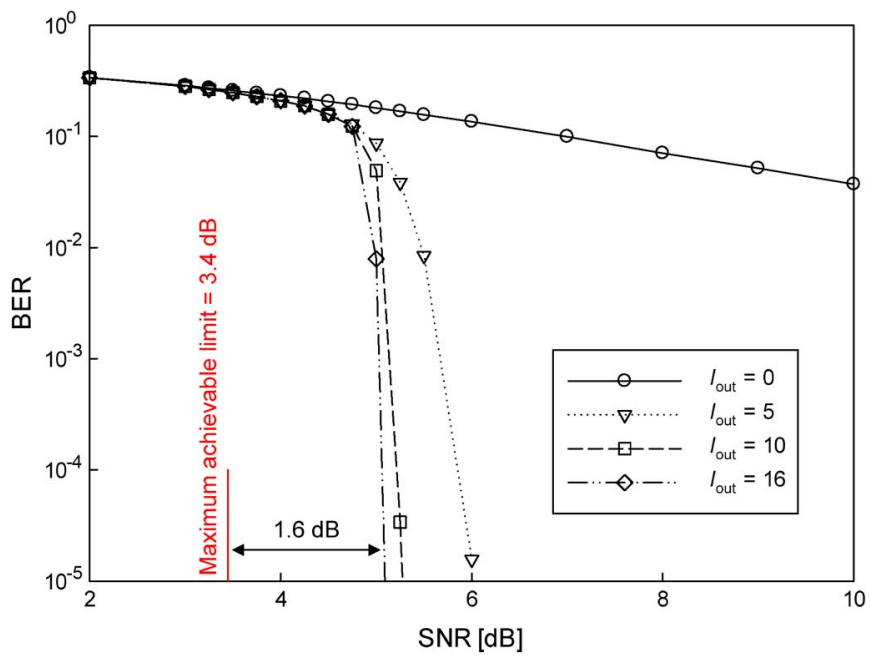

Fig. 5. Achievable BER curves of our FDE-aided SC-SM system, employing $M=N=4$ transmit and receive antenna elements and 16-PSK modulation. The normalized transmission rate of the half-rate channel-encoded system was $R=3 \mathrm{bps} / \mathrm{Hz}$. The interleaver length was $38400 \mathrm{bits}$, we used the half-rate $\operatorname{RSC}(2,1,2)$ code, and the block length was $K=256$.

351 channels. Hence, a novel SoD FDE algorithm was developed for our 352 SM-MIMO scheme. This algorithm enables us to operate in a realistic 353 dispersive fading channel exhibiting a long CIR while attaining a near354 capacity performance. shift keying: A dispersion matrix approach," IEEE Trans. Commun., vol. 58, no. 11, pp. 3219-3230, Nov. 2010.
[10] M. Di Renzo and H. Haas, "A general framework for performance 382 analysis of space shift keying (SSK) modulation for MISO correlated 383 Nakagami-m fading channels," IEEE Trans. Commun., vol. 58, no. 9, 384 pp. 2590-2603, Sep. 2010.

[11] S. Sugiura and L. Hanzo, "Effects of channel estimation on spatial 386 modulation," IEEE Signal Process. Lett., vol. 19, no. 12, pp. 805-808, 387 Dec. 2012.

[12] S. Ganesan, R. Mesleh, H. Haas, C. W. Ahn, and S. Yun, "On the per- 389 formance of spatial modulation OFDM," in Proc. 40th Asilomar Conf. 390 Signals, Syst. Comput., 2006, pp. 1825-1829.

[13] R. Y. Mesleh, H. Haas, S. Sinanovic, C. Ahn, and S. Yun, "Spatial 392 modulation," IEEE Trans. Veh. Technol., vol. 57, no. 4, pp. 2228-2242, 393 Jul. 2008.

[14] M. Kadir, S. Sugiura, J. Zhang, S. Chen, and L. Hanzo, "OFDMA/SC- 395 FDMA aided space-time shift keying for dispersive multiuser scenarios," 396 IEEE Trans. Veh. Technol., vol. 62, no. 1, pp. 408-414, Jan. 2013.

[15] A. Kalis, A. G. Kanatas, and C. B. Papadias, "A novel approach to MIMO 398 transmission using a single RF front end," IEEE J. Sel. Areas Commun., 399 vol. 26, no. 6, pp. 972-980, Aug. 2008.

[16] O. N. Alrabadi, J. Perruisseau-Carrier, and A. Kalis, "MIMO transmission 401 using a single RF source: Theory and antenna design," IEEE Trans. 402 Antennas Propag., vol. 60, no. 2, pp. 654-664, Feb. 2012. virtual MIMO systems," IEEE Signal Process. Lett., vol. 21, no. 4, 405 pp. 390-394, Apr. 2014.

[18] H. A. Ngo, C. Xu, S. Sugiura, and L. Hanzo, "Space-time-frequency 407 shift keying for dispersive channels," IEEE Signal Process. Lett., vol. 18, 408 no. 3, pp. 177-180, Mar. 2011.

[19] P. Som and A. Chockalingam "Spatial modulation and space shift keying 410 in single carrier communication," in Proc. IEEE 23rd Int. Symp. PIMRC, 411 Sep. 2012, pp. 1962-1967.

[20] R. Rajashekar, K. V. S. Hari, and L. Hanzo, "Spatial modulation aided 413 zero-padded single carrier transmission for dispersive channels," IEEE 414 Trans. Commun., vol. 61, no. 6, pp. 2318-2329, Jun. 2013.

[21] F. Pancaldi, G. Vitetta, R. Kalbasi, N. Al-Dhahir, M. Uysal, and 416 H. Mheidat, "Single-carrier frequency domain equalization," IEEE Signal 417 Process. Mag., vol. 25, no. 5, pp. 37-56, Sep. 2008.

[22] K. Ishibashi and S. Sugiura, "Effects of antenna switching on band- 419 limited spatial modulation," IEEE Wireless Commun. Lett., vol. 3, no.4, 420 pp. 345-348, Aug. 2014.

[23] L. Hanzo, T. Liew, B. Yeap, R. Y. S. Tee, and S. X. Ng, Turbo Coding, 422 Turbo Equalisation, and Space-Time Coding for Transmission over Fad- 423 ing Channels. Hoboken, NJ, USA: Wiley, 2011.

[24] S. ten Brink, "Convergence behavior of iteratively decoded parallel con- 425 catenated codes," IEEE Trans. Commun., vol. 49, no. 10, pp. 1727-1737, 426 Oct. 2001.

[25] L. Hanzo, L.-L. Yang, E.-L. Kuan, and K. Yen, Single and Multi-Carrier 428 CDMA: Multi-User Detection, Space-Time Spreading, Synchronisation 429 and Standards, Hoboken, NJ, USA: Wiley, 2003.

430

[26] M. Tüchler, A. Singer, and R. Koetter, "Minimum mean squared error 431 equalization using a priori information," IEEE Trans. Signal Process., 432 vol. 50, no. 3, pp. 673-683, Mar. 2002.

[27] S. Sugiura, S. Chen, and L. Hanzo, "MIMO-aided near-capacity turbo 434 transceivers: Taxonomy and performance versus complexity," IEEE 435 Commun. Surveys Tuts., vol. 14, no. 2, pp. 421-442, May 2012.

[28] L. Hanzo, O. Alamri, M. El-Hajjar, and N. Wu, Near-Capacity Multi- 437 Functional MIMO Systems: Sphere-Packing, Iterative Detection and 438 Cooperation. Hoboken, NJ, USA: Wiley, 2009.

[29] S. ten Brink, "Designing iterative decoding schemes with the extrinsic 440 information transfer chart," AEU Int. J. Electron. Commun., vol. 54, 441 no. 6, pp. 389-398, Nov. 2000.

[30] S. ten Brink, G. Kramer, and A. Ashikhmin, "Design of low-density 443 parity-check codes for modulation and detection," IEEE Trans. Commun., 444 vol. 52, no. 4, pp. 670-678, Apr. 2004. 


\section{AUTHOR QUERIES}

AUTHOR PLEASE ANSWER ALL QUERIES

AQ1 = Please provide keywords.

END OF ALL QUERIES 


\section{Correspondence}

\section{Single-RF Spatial Modulation Requires Single-Carrier \\ 2 Transmission: Frequency-Domain Turbo \\ 3 Equalization for Dispersive Channels}

4

5

\author{
Shinya Sugiura, Senior Member, IEEE, and \\ Lajos Hanzo, Fellow, IEEE
}

6 Abstract-In this paper, we propose a broadband single-carrier (SC) 7 spatial modulation (SM)-based multiple-input-multiple-output (MIMO) 8 architecture relying on a soft decision (SoD) frequency-domain equal9 ization (FDE) receiver. We demonstrate that conventional orthogonal-

10 frequency-division-multiplexing (OFDM)-based broadband transmissions

11 are not readily suitable for the single-radio-frequency-assisted SM-MIMO

12 schemes since this scheme exhibits no substantial performance advantage

13 over single-antenna transmissions. To circumvent this limitation, a low-

14 complexity SoD FDE algorithm based on the minimum mean square error

15 (MMSE) criterion is invoked for our broadband SC-based SM-MIMO

16 scheme, which is capable of operating in a strongly dispersive channel

17 having a long channel impulse response at moderate decoding complexity.

18 Furthermore, our SoD FDE attains a near-capacity performance with the

19 aid of a three-stage concatenated SC-based SM architecture.

20 Index Terms-Author, please supply index terms/keywords for your 22 paper. To download the IEEE Taxonomy go to http://www.ieee.org/ documents/taxonomy_v101.pdf.

\section{INTRODUCTION}

24 Spatial modulation (SM)-based multiple-input-multiple-output 25 (MIMO) designs have become popular as a benefit of their low-cost 26 single radio frequency (RF) transmitters and their ability to increase 27 the attainable transmission rates [1]-[3]. The information bits of the $28 \mathrm{SM}$ transmitter are mapped to both the spatial (antenna) dimension 29 and to the classic amplitude phase-shift keying (APSK) constellation. 30 More specifically, one of the $M$ transmit antenna (TA) elements 31 is activated by $\log _{2} M$ information bits, whereas a complex-valued 32 APSK symbol $s_{l}$, which is constituted by $\log _{2} \mathcal{L}$ information bits, is 33 transmitted from the activated TA. Hence, a total of $B=\log _{2}(\mathcal{L} \cdot M)$ 34 bits are conveyed during each symbol interval by using a single-RF35 based transmitter.

36 Current wireless telecommunication standards typically employ 37 broadband techniques [4], such as orthogonal frequency-division mul38 tiplexing (OFDM) [5] and single-carrier (SC) frequency-division mul39 tiple access [6]. However, the majority of previous SM studies has 40 focused on narrowband scenarios, assuming that the SM symbols are 41 transmitted over a frequency-flat channel [7]-[11].

42 Nevertheless, some OFDM-based broadband SM schemes have 43 also been developed [12], [13]; these are, however, less attractive

Manuscript received June 24, 2014; revised October 2, 2014; accepted November 11, 2014. This work was supported by the Japan Society for the Promotion of Science Grants-in Aid for Scientific Research (KAKENHI) under Grant 26709028. The review of this paper was coordinated by Dr. C. Cozzo.

S. Sugiura is with the Department of Computer and Information Sciences, Tokyo University of Agriculture and Technology, Tokyo 184-8588, Japan (e-mail: sugiura@ieee.org).

L. Hanzo is with the School of Electronics and Computer Science,University of Southampton, Southampton SO17 1BJ, U.K. (e-mail: 1h@ecs.soton.ac.uk).

Color versions of one or more of the figures in this paper are available online at http://ieeexplore.iee.org.

Digital Object Identifier 10.1109/TVT.2014.2370679 from a practical point of view, although this has not been explicitly 44 detailed before. For instance, let us assume that the SM scheme's TA 45 activation process is individually implemented for each subcarrier of 46 an OFDM system. This requires that multiple TA elements have to be 47 simultaneously activated over the OFDM frame, hence precluding the 48 benefit of having the abovementioned single-RF-based SM scheme. 49 In practice, to maintain a single-RF SM transmitter structure, the 50 previously proposed OFDM-based SM schemes [12], [13] have to 51 rely on block-based antenna activation, in which the TA activation 52 process is carried out for each OFDM frame, rather than for each 53 subcarrier. ${ }^{1}$ In this architecture, the SM scheme's contribution to the 54 rate increase per subcarrier becomes as low as $\left(\log _{2} M\right) / N_{C}$, where 55 $N_{C}$ is the number of subcarriers. This gain is $N_{C}$ times lower than 56 that expected in a narrowband SM-MIMO scenario. In this sense, the 57 OFDM-based SM scheme's advantage over the conventional single- 58 antenna-aided system is negligible for a practical broadband scenario, 59 in which hundreds of subcarriers are supported. In general, the same 60 holds not only for the SM scheme but also for most of the MIMO 61 schemes relying on a single-RF transmitter [15]-[17]. However, this 62 issue has not been explicitly considered, in spite of its significant 63 importance in terms of realistic broadband communications. ${ }^{2} \quad 64$

The broadband SC-based SM architecture has the potential of 65 solving the problems of the abovementioned OFDM-based SM-MIMO 66 schemes. Since the SM scheme's TA activation process is carried out 67 for each symbol in the SC-based SM architecture, the benefits of an 68 increased transmission rate and a low-cost single-RF transmitter are 69 maintained, while facilitating its operation as a broadband system. 70 So far, only very few SC-based SM schemes capable of operating 71 in dispersive channels have been developed [18]-[20]. In [18], the 72 SM scheme's TA activation concept was combined with frequency- 73 shift keying modulation, which spreads the transmitted signal not 74 only across the spatial domain but across the frequency domain (FD) 75 as well. In [19], a cyclic prefix (CP)-based SC-MIMO scheme was 76 developed, which relied on exhaustive maximum likelihood (ML) 77 detection. In [20], zero padding (ZP)-aided SC-SM schemes based on 78 time domain (TD) ML equalization and reduced-complexity parallel- 79 interference cancelation were proposed to achieve the maximum at- 80 tainable transmit and receive diversity gains. However, the frame 81 length and the channel impulse response (CIR) length considered in 82 [18]-[20] was less than ten taps, although the CIR length of practical 83 broadband channels is often significantly higher. More importantly, 84 all the previous SC-SM schemes [18]-[20] were developed for hard- 85 decision-based receivers, which prevents us from exploiting the bene- 86 fits of powerful iterative detection.

To eliminate the effects of long CIRs encountered in practical 88 broadband dispersive channels, an efficient equalization algorithm has 89 to be conceived for the SC-SM scheme. Furthermore, to employ a 90

\footnotetext{
${ }^{1}$ When considering a full-RF SM-MIMO transmitter that is equipped with the same number of RF chains as that of the TA elements, as shown in [14], the subcarrier-based OFDM-SM system is capable of operating without imposing a penalty on the transmission rate. However, such a full-RF transmitter imposes a higher terminal cost than its single-RF counterpart.

${ }^{2}$ To provide further insights, studies of conventional single-RF MIMO schemes have focused, for simplicity, on narrowband scenarios associated with frequency-flat fading. However, unlike for its full-RF MIMO counterparts, its application to broadband transmissions is not straightforward. This challenge is tackled in this paper.
} 
TABLE I

Fundamental COMPARISONS BETWEen THE BROADBAND SM-MIMO SCHEMES

\begin{tabular}{|c|c|c|c|}
\hline$\overline{\overline{\text { Type }}}$ & Normalized transmission rate $[\mathrm{bps} / \mathrm{Hz}]$ & Number of RF chains & Number of transmit antennas \\
\hline Proposed SC-based SM-MIMO & $\log _{2}(M \cdot \mathcal{L})$ & 1 & $M$ \\
\hline Subcarrier-based OFDM-SM-MIMO & $\log _{2}(M \cdot \mathcal{L})$ & $M$ & $M$ \\
\hline 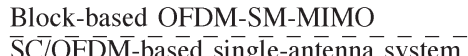 & $\log _{2}(\mathcal{L})+\left(\log _{2} M\right) / N_{C}$ & -1 & $\frac{M}{1}$ \\
\hline SC/OFDM-based single-antenna system & & & \\
\hline
\end{tabular}

91 powerful channel-coding scheme relying on iterative detection, the 92 SC-SM detector has to output soft information. In the context of classic 93 single-antenna-based or MIMO arrangements, an efficient soft deci94 sion (SoD) frequency-domain equalization (FDE) was proposed and 95 standardized for the Long-Term Evolution system [21]. To the best of 96 our knowledge, an efficient SoD equalization algorithm that is capable 97 of exchanging extrinsic information with a powerful channel-coding 98 scheme relying on soft-input soft-output (SISO) iterative detection has 99 not been conceived for a broadband SC-based SM scheme. ${ }^{3}$

100 Against this background, the novel contributions of this paper are as 101 follows.

103

104

105

106

107

108

109

110

111

112

113

114

115

116

117

118

119

120

121

122 The remainder of this paper is organized as follows. In Section II, we 123 describe the model of our broadband SC-based SM scheme, whereas in 124 Section III, we present our FDE algorithm. In Section IV, the proposed 125 scheme's iterative convergence behavior and maximum achievable 126 limit are analyzed. In Section V, we consider the performance of our 127 system, whereas our conclusions are presented in Section VI.

\section{SYSTEM MODEL}

129 Here, we commence by clarifying our motivation of designing an 130 SC-based SM-MIMO system, rather than its OFDM-based counter131 part. Then, we outline the model of our SC-based SM-MIMO system.

\section{A. Preliminary Discussions of Our Broadband 133 SM-MIMO Scheme}

134 Before detailing the proposed SC-based SM-MIMO system, we 135 introduce the broadband SM-MIMO family and analyze the limitations 136 imposed on the previous OFDM-based SM-MIMO system.

\footnotetext{
${ }^{3}$ Assuming single-RF SM-MIMO transmissions, the SM-specific shaping filter has to be designed so that the pulse is isolated in the TD. This may reduce the bandwidth efficiency and the power amplifier efficiency in comparison with a classic modem employing an efficient raised-cosine filter. However, this issue is beyond the scope of this paper; the details are discussed in [22].
}

The bandwidth efficiency of a conventional OFDM- or SC-based 137 single-antenna system is given by

$$
R_{\text {SISO }}=\log _{2} \mathcal{L}[\mathrm{bps} / \mathrm{Hz}]
$$

where $\mathcal{L}$ is the constellation size. For simplicity, we assume that the 139 relative overhead of the guard interval or CP over the frame length is 140 sufficiently low.

Next, let us consider the OFDM-based SM-MIMO scheme relying 142 on a single-RF transmitter. As briefly mentioned in the introduction, 143 a single-RF transmitter is unable to simultaneously activate multiple 144 TA elements. Hence, the entire OFDM frame, including the $N_{\mathrm{C}} 145$ $\mathcal{L}$-PSK/quadrature amplitude modulation (QAM)-based subcarriers, 146 must be transmitted by a single activated TA element. The bandwidth 147 efficiency of the OFDM-based SM-MIMO system is

$$
R_{\mathrm{SM}-\mathrm{MIMO}}^{\text {(OFD) }}=\log _{2} \mathcal{L}+\frac{\log _{2} M}{N_{\mathrm{C}}}[\mathrm{bps} / \mathrm{Hz}]
$$

where the first term represents the classic $\mathcal{L}$-point APSK-modulated 149 subcarrier, whereas the second term corresponds to the TA activation 150 process carried out per OFDM frame. Note that $R_{\text {SM-MIMO }}^{\text {(OFDM }}$ in (2) 151 increases to $\log _{2}(M \cdot \mathcal{L})$ in the full-RF SM-MIMO transmitter, since 152 the SM scheme's antenna activation process is carried out for each 153 subcarrier. However, this is achieved at the price of increasing the 154 transmitter's cost, hence relinquishing the main benefit of the single- 155 RF SM scheme.

By contrast, the SC-based SM-MIMO architecture facilitates the 157 SM scheme's independent TA activation process for each symbol, i.e., 158 one of the $M$ TA elements is activated during each symbol interval, 159 and the $\mathcal{L}$-size PSK/QAM symbol is transmitted from the activated 160 TA. Therefore, the bandwidth efficiency of the SC-based SM-MIMO 161 system becomes 162

$$
R_{\mathrm{SM}-\mathrm{MIMO}}^{\mathrm{SC}}=\log _{2} \mathcal{L}+\log _{2} M[\mathrm{bps} / \mathrm{Hz}]
$$

which reflects the expected throughput gain of the SM scheme [2], 163 [3]. However, note again that this is not attainable by the OFDM- 164 based single-RF SM architecture represented by (2). The fundamental 165 comparisons between the various broadband SM-MIMO schemes are 166 shown in Table I and Fig. 1.

\section{B. Model of Our SC-Based SM-MIMO Scheme}

Let us consider a broadband SC-SM transmitter having $M$ TAs 169 and using an $\mathcal{L}$-sized PSK/QAM modulation scheme. Similar to the 170 narrowband SM scheme, each SM symbol contains $B_{1}=\log _{2} M 171$ and $B_{2}=\log _{2} \mathcal{L}$ information bits, where one of the $M$ TAs is acti- 172 vated according to $B_{1}$ bits, whereas the $B_{2}$ bits are mapped onto a 173 PSK/QAM symbol $s_{l}(k)$. Furthermore, $k$ is the symbol index, and we 174 denote the index of the activated TA during the $k$ th interval by $m(k) .175$ For simplicity, we employ a vectorial notation for the SM symbol, as 176 follows:

$$
\mathbf{s}(k)=[\underbrace{0, \ldots, 0}_{m(k)-1}, s_{l}(k), \underbrace{0, \ldots, 0}_{M-m(k)}]^{T} \in \mathbb{C}^{M \times 1} .
$$




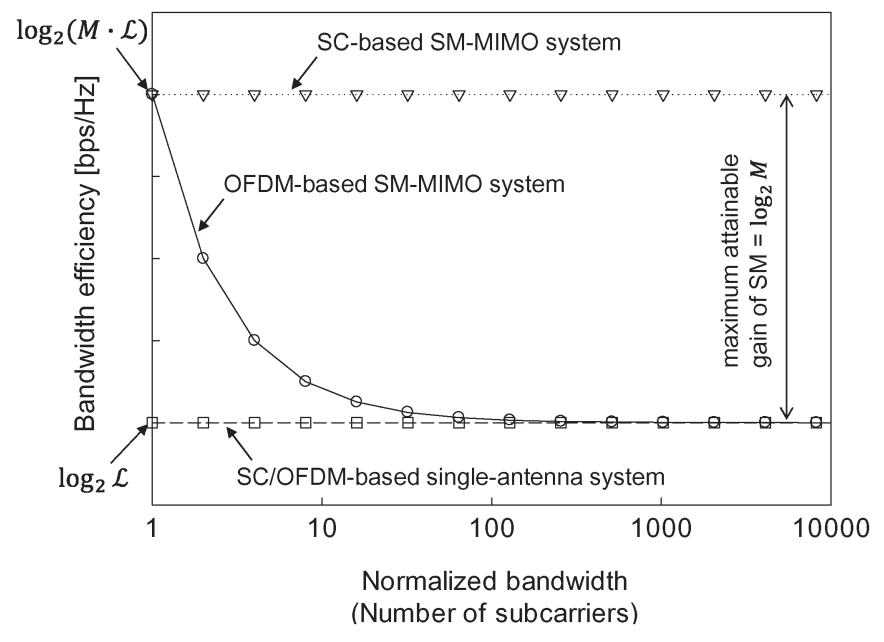

Fig. 1. Bandwidth efficiencies of the broadband OFDM- and SC-based SM-MIMO systems; that of the classic SC/OFDM-based single-antenna system is included as a benchmark.

178 We consider block transmissions of the $K$ SM symbols, i.e.,

$$
\mathbf{S}=[\mathbf{s}(1), \ldots, \mathbf{s}(K)]^{T} \in \mathbb{C}^{M \times K} .
$$

179 After concatenating the $\nu$-length $\mathrm{CP}$, which is higher than the CIR 180 length $\xi$, the SM symbol block is transmitted over $(K+\nu)$ consecutive 181 symbol durations.

182 At the receiver, the $\nu$-length $\mathrm{CP}$ is removed from the received $(K+$ $183 \nu$ )-length SM block. Then, we arrive at

$$
\begin{aligned}
\mathbf{y}= & {\left[y_{1}(1), \ldots, y_{1}(K), \ldots\right.} \\
& \left.y_{N}(1), \ldots, y_{N}(K)\right]^{T} \in \mathbb{C}^{N K \times 1} \\
= & \mathbf{H} \overline{\mathbf{s}}+\mathbf{n}
\end{aligned}
$$

184 where $\overline{\mathbf{s}} \in \mathbb{C}^{M K \times 1}$ is given by a vector stacking operation applied to $185 \mathrm{~S}$. Furthermore, $N$ is the number of receive antenna elements, whereas $186 \mathbf{n} \in \mathbb{C}^{N \times 1}$ denotes the associated additive noise components, where 187 the random variables are distributed according to the complex-valued 188 Gaussian distribution $\mathcal{C N}\left(0, N_{0}\right)$, with zero mean and variance $N_{0}$. 189 Moreover, the channel components $\mathbf{H} \in \mathbb{C}^{N K \times M K}$ are expressed as 190 submatrices, as follows:

$$
\mathbf{H}=\left[\begin{array}{ccc}
\mathbf{H}_{11} & \cdots & \mathbf{H}_{1 M} \\
\vdots & \ddots & \vdots \\
\mathbf{H}_{N 1} & \cdots & \mathbf{H}_{N M}
\end{array}\right]
$$

191 where each submatrix $\mathbf{H}_{n m} \in \mathbb{C}^{K \times K}$ represents a circular matrix, 192 which is composed of the $\xi$-length CIRs $\mathbf{h}_{n m}=\left[h_{n m}^{(1)}, \ldots, h_{n m}^{(\xi)}\right]^{T} \in$ $193 \mathbb{C}^{\xi \times 1}$, while assuming the relationship of $\xi \leq \nu<K$.

\section{III. FREQUENCY DOMAIN EQUALIZATION-AIDED \\ 195 SINGLE-CARRIER-SPATIAL MOdULATION 196 Multiple-InPut-Multiple-OutPut ReCEIVER}

197 Here, we derive our hard-decision SC-SM FDE receiver and then 198 extend it to its SoD counterpart, which is suitable for iterative detection 199 and is based on the turbo principle [23].

\section{A. Hard-Decision SC-SM Receiver}

201 With the aid of fast Fourier transforms (FFTs), each channel subma202 trix $\mathbf{H}_{n m}$ is represented by

$$
\mathbf{H}_{n m}=\mathbf{Q}^{T} \boldsymbol{\Lambda}_{n m} \mathbf{Q}^{*}
$$

where the element in the $k$ th row and $l$ th column of $\mathbf{Q}$ is 203 given by $[\mathbf{Q}]_{k l}=(1 / \sqrt{K}) \exp [-2 \pi j(k-1)(l-1) / K]$. Further- 204 more, $\Lambda_{n m} \in \mathbb{C}^{K \times K}$ denotes the diagonal matrix for which the 205 nonzero elements are the $K$ FFT coefficients. Hence, the received 206 signals of (7) can be rewritten as

$$
\begin{aligned}
\mathbf{y} & =\left(\mathbf{I}_{N} \otimes \mathbf{Q}^{T}\right) \mathbf{\Lambda}\left(\mathbf{I}_{\mathbf{M}} \otimes \mathbf{Q}^{*}\right) \overline{\mathbf{s}}+\mathbf{n} \\
& =\left(\mathbf{I}_{N} \otimes \mathbf{Q}^{T}\right) \mathbf{\Lambda} \mathbf{s}_{f}+\mathbf{n}
\end{aligned}
$$

where we have

$$
\begin{aligned}
\mathbf{\Lambda} & =\left[\begin{array}{ccc}
\boldsymbol{\Lambda}_{11} & \cdots & \boldsymbol{\Lambda}_{1 M} \\
\vdots & \ddots & \vdots \\
\boldsymbol{\Lambda}_{N 1} & \cdots & \boldsymbol{\Lambda}_{N M}
\end{array}\right] \in \mathbb{C}^{N K \times M K} \\
\mathbf{s}_{f} & =\left(\mathbf{I}_{\mathbf{M}} \otimes \mathbf{Q}^{*}\right) \overline{\mathbf{s}} \in \mathbb{C}^{M K \times 1} .
\end{aligned}
$$

Moreover, $\mathbf{I}_{n} \in \mathbb{R}^{n \times n}$ is the $n$-size identity matrix, and $\otimes$ represents 209 the Kronecker product.

Upon multiplying both sides of (11) by $\left(\mathbf{I}_{N} \otimes \mathbf{Q}^{*}\right)$, we arrive at the 211 received signals $\mathbf{y}_{f}$ in the $\mathrm{FD}$, as follows:

$$
\mathbf{y}_{f}=\Lambda \mathbf{s}_{f}+\mathbf{n}_{f}
$$

where $\mathbf{n}_{f}=\left(\mathbf{I}_{N} \otimes \mathbf{Q}^{*}\right) \mathbf{n}$. Next, MMSE filtering is invoked for es- 213 timating the FD SC-SM signals $\mathbf{s}_{f}$ by minimizing the average MSE 214 between the FD SM symbols $\mathbf{s}_{f}$ and the estimates $\hat{\mathbf{s}}_{f}$. Given the 215 complex-valued weights $\mathbf{w} \in \mathbb{C}^{N K \times 1}$, the MMSE-filtered outputs are 216 given by

$$
\hat{\mathbf{s}}_{f}=\mathbf{w}^{T} \mathbf{y}_{f} .
$$

According to [25], the complex-valued MMSE equalizer weights w 218 are calculated as follows:

$$
\begin{aligned}
\mathbf{w} & =\left(\mathbf{R}_{y y}\right)^{-1} \mathbf{R}_{y s} \\
& =\left(\frac{\boldsymbol{\Lambda} \boldsymbol{\Lambda}^{H}}{M}+N_{0} \mathbf{I}_{N K}\right)^{-1} \frac{\boldsymbol{\Lambda}}{M}
\end{aligned}
$$

where we have

$$
\begin{aligned}
& \mathbf{R}_{y y}=\mathbb{E}\left[\mathbf{y}_{f} \mathbf{y}_{f}^{H}\right]=\frac{\boldsymbol{\Lambda} \boldsymbol{\Lambda}^{H}}{M}+N_{0} \mathbf{I}_{N K} \\
& \mathbf{R}_{y s}=\mathbb{E}\left[\mathbf{y}_{f} \mathbf{s}_{f}^{H}\right]=\frac{\boldsymbol{\Lambda}}{M}
\end{aligned}
$$

while

$$
\begin{aligned}
\mathbb{E}\left[\mathbf{s}_{f} \mathbf{s}_{f}^{H}\right] & =\mathbb{E}\left[\left(\mathbf{I}_{\mathbf{M}} \otimes \mathbf{Q}^{*}\right) \overline{\mathbf{s}} \overline{\mathbf{s}}^{H}\left(\mathbf{I}_{\mathbf{M}} \otimes \mathbf{Q}^{T}\right)\right] \\
& =\frac{\mathbf{I}_{M K}}{M} .
\end{aligned}
$$

Note that in the terms that include the coefficient $M, \mathbf{R}_{y y}$, and $\mathbf{R}_{y s} 222$ of (18) and (19) are different from those derived for conventional 223 equalization or for the traditional MIMO systems. This is because the 224 SM symbol $\mathbf{s}(k)$ contains only a single nonzero element and because 225 the sparsity factor of $\overline{\mathbf{s}}$ is $M$, as shown in (20).

Next, we convert the FD estimates $\hat{\mathbf{s}}_{f}$ of (15) into their TD counter- 227 parts, as follows:

$$
\hat{\overline{\mathbf{s}}}=\left(\mathbf{I}_{M} \otimes \mathbf{Q}^{T}\right) \hat{\mathbf{s}}_{f} .
$$

By rearranging the vector $\hat{\overline{\mathbf{s}}}$, we arrive at the SC-SM estimates of

$$
\hat{\mathbf{S}}=[\hat{\mathbf{s}}(1), \ldots, \hat{\mathbf{s}}(K)]^{T}
$$

which corresponds to the transmitted SM frame $\mathbf{S}$ shown in (5). 


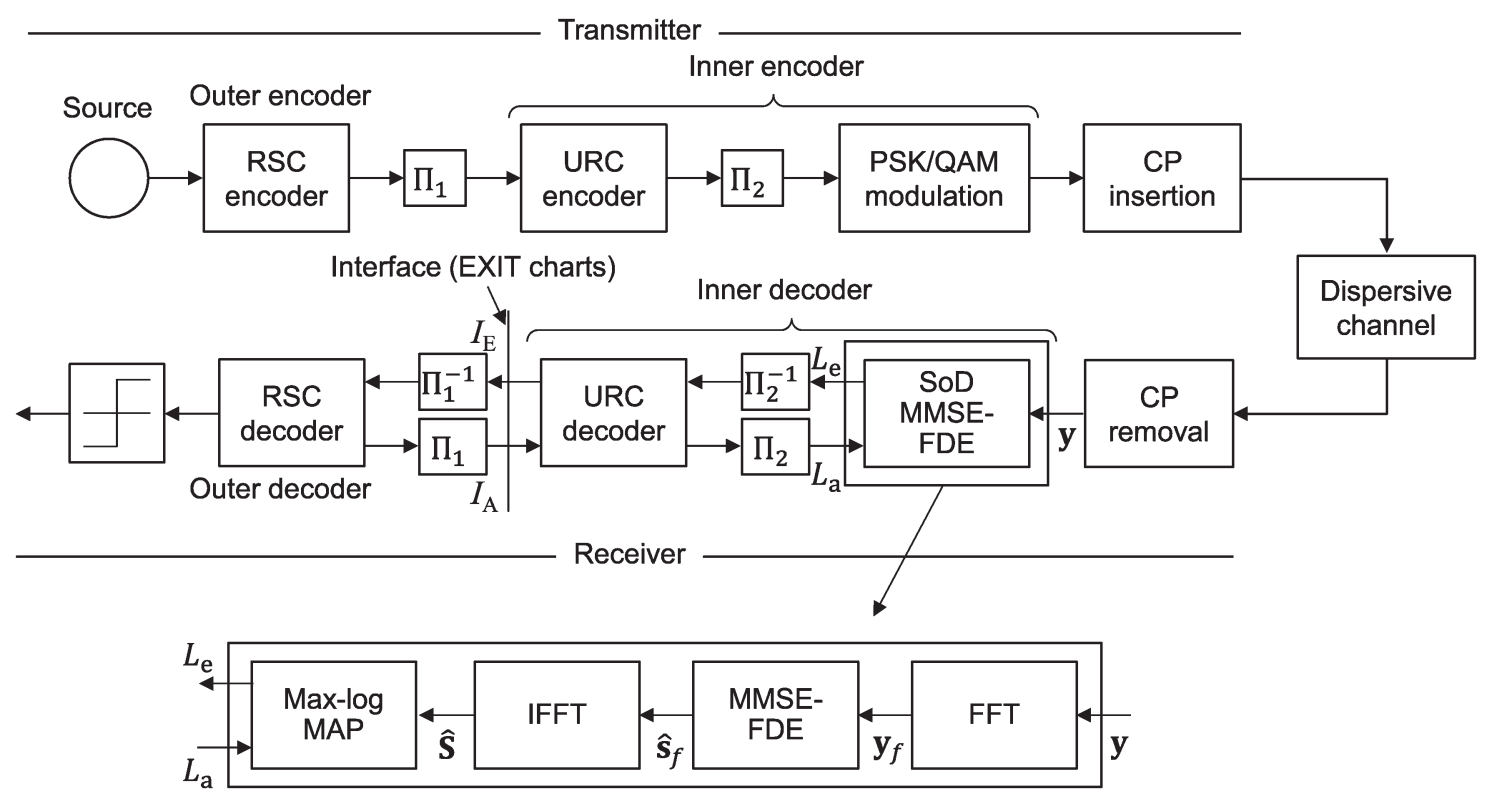

Fig. 2. Transceiver architecture of our broadband SC-based SM scheme.

231 Finally, symbol-based ML detection is applied to $\hat{\mathbf{S}}$

$$
\langle\hat{m}(k), \hat{l}(k)\rangle=\arg \min _{m, l}\left\|\hat{\mathbf{s}}(k)-\mathbf{s}_{m, l}\right\|^{2}
$$

232 where we have

$$
\mathbf{s}_{m, l}=[\underbrace{0, \ldots, 0}_{m-1}, s_{l}, \underbrace{0, \ldots, 0}_{M-m}]^{T} \in \mathbb{C}^{M \times 1} .
$$

233 Note that (23) represents symbol-by-symbol ML detection, which is 234 equivalent to additive white Gaussian noise, and hence, it is indepen235 dent both of the CIR length $\xi$ as well as of the frame length $K$. This 236 allows us to benefit from the SM scheme's low decoding complexity.

\section{B. SoD SC-SM Receiver}

238 Here, we extend the hard-decision SC-SM receiver derived in the 239 previous section to its SoD version. Typically, the MMSE-based SoD 240 MIMO receiver employs the soft-interference cancelation concept 241 proposed in [26]. However, in our SC-SM scheme, it is a challenging 242 task to compute soft SM symbols from the a priori information, due to 243 the SM-specific TA activation principle. ${ }^{4}$

244 Instead of the hard-decision ML detection of (23), we simply carry 245 out SoD maximum a posteriori (MAP) demodulation. By using the 246 intersymbol-interference-free estimates of the SM symbol vector $\hat{\mathbf{s}}(k)$ 247 shown in (22), we arrive at the extrinsic log-likelihood ratio (LLR) 248 value of the bit $b_{p}\left(p=1, \ldots, \log _{2}(M \cdot \mathcal{L})\right)$, which is included in the $249 k$ th SM symbol, as follows [9]:

$$
\begin{aligned}
L_{\mathrm{e}}\left(b_{p}\right)= & \max _{\mathbf{s}_{m, l} \in \mathbf{s}_{1}^{p}}\left[-\frac{\left\|\hat{\mathbf{s}}(k)-\mathbf{s}_{m, l}\right\|}{N_{\mathrm{MAP}}}+\sum_{j \neq k} b_{j} L_{\mathrm{a}}\left(b_{j}\right)\right] \\
& -\max _{\mathbf{s}_{m, l} \in \mathbf{s}_{0}^{p}}\left[-\frac{\left\|\hat{\mathbf{s}}(k)-\mathbf{s}_{m, l}\right\|}{N_{\mathrm{MAP}}}+\sum_{j \neq k} b_{j} L_{\mathrm{a}}\left(b_{j}\right)\right]
\end{aligned}
$$

\footnotetext{
${ }^{4}$ To expound further, since the SM mapping scheme attained by antenna activation is discrete, it is difficult to define the soft values.
}

where $\mathbf{s}_{1}^{p}$ and $\mathbf{s}_{0}^{p}$ represent the subspace of the legitimate equiva- 250 lent signals, satisfying $\mathbf{s}_{1}^{p} \equiv\left\{\mathbf{s}_{m, l} \in \mathbf{s}: b_{p}=1\right\}$ and $\mathbf{s}_{0}^{p} \equiv\left\{\mathbf{s}_{m, l} \in 251\right.$ $\left.\mathbf{s}: b_{p}=0\right\}$, respectively. Furthermore, $L_{\mathrm{a}}(\cdot)$ represents the a priori 252 information expressed in terms of LLRs, whereas $N_{\text {MAP }}$ denotes the 253 variance of the noise that was included in the SM symbol estimates 254 $\hat{\mathbf{s}}(k)$. Since the SoD demodulation of (25) is based on a symbol-by- 255 symbol operation similar to the hard-decision version of (23), low 256 complexity is maintained.

\section{EXIT-Chart-Aided SEMIANALYSis OF OUR FREQUENCY DOMAIN EQUALIZATION-AIDED Single-CARRIER-SPATIAL-MOdULATION SCHEME}

\section{A. Three-Stage Concatenated SC-SM Transceiver}

Fig. 2 shows our three-stage concatenated recursive systematic 263 convolutional (RSC)-coded and unity-rate convolutional (URC)-coded 264 SC-SM structures. The transmitter channel encodes the source infor- 265 mation bits using the RSC code, and these are then interleaved by the 266 first interleaver $\Pi_{1}$. The interleaved bits are then encoded by the URC 267 code, and these are then interleaved again by the interleaver $\Pi_{2}$. The 268 resultant bits are then mapped to the SC-SM symbols S. After adding 269 the $\mathrm{CP}$ symbols to $\mathbf{S}$, the $\mathrm{SM}$ symbols are transmitted.

As shown in Fig. 1, at the receiver, the CP symbols are removed 271 from the received signal block. Next, the SISO decoders (i.e., the SoD 272 FDE-aided SC-SM decoder proposed in Section III, the URC decoder, 273 and the RSC decoder) iteratively exchange their extrinsic information. 274 For each of the $I_{\text {out }}$ outer iterations, there are $I_{\text {in }}$ inner iterations carried 275 out between each SC-SM decoder and the associated URC decoder. 276 Therefore, the total number of iterations is $\left(I_{\text {in }} \cdot I_{\text {out }}\right)$. The details of 277 the three-stage concatenated system can be found in [27] and [28]. 278

\section{B. Convergence Behavior Analysis}

Here, we use EXIT charts [24] for visualizing the convergence 280 behavior of the iterative detection. We present the EXIT charts of our 281 SC-based SM scheme, where $M=N=4$ TAs and receive antennas 282 were used, whereas the signal-to-noise ratio (SNR) was varied from 283 0 to $10 \mathrm{~dB}$, in steps of $1 \mathrm{~dB}$. The outer code's EXIT curve is 284 also plotted for the half-rate $\operatorname{RSC}(2,1,2)$ code, having the octally 285 


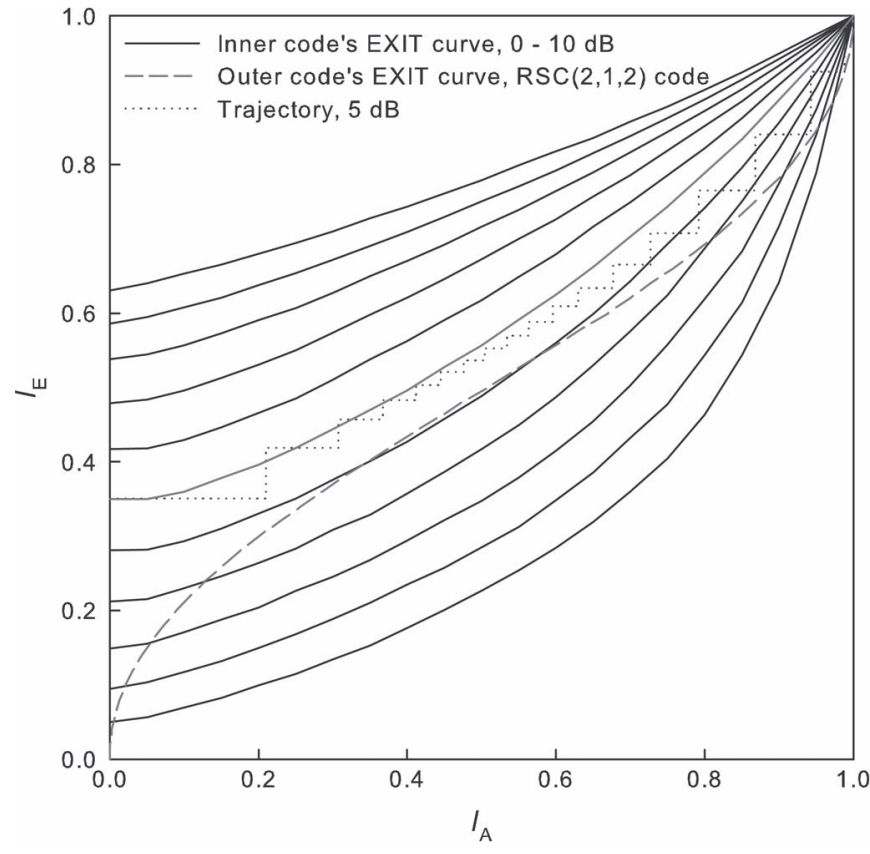

Fig. 3. EXIT charts of our MMSE-FDE-aided SC-based SM-MIMO system, employing $M=N=4$ transmit and receive antenna elements and 16-PSK modulation. The normalized transmission rate of the half-rate channel-encoded system was $R=3 \mathrm{bps} / \mathrm{Hz}$. The EXIT trajectory was calculated by assuming that the code length was 38400 bits and that the $\mathrm{SNR}=5 \mathrm{~dB}$.

286 represented generator polynomials of $\left(G_{\mathrm{r}}, G\right)=(3,2)$ [29], where $287 G_{\mathrm{r}}$ is the recursive feedback polynomial, and $G$ is the feedforward 288 polynomial. We assumed frequency-selective Rayleigh fading with 289 equal-power 15-length CIRs. Furthermore, an $(\mathcal{L}=16)$-PSK modu290 lation scheme was considered, and the normalized transmission rate 291 of the half-rate channel-encoded system was $R=3 \mathrm{bps} / \mathrm{Hz}$. The 292 EXIT trajectory was calculated by assuming that the code length was 29338400 bits, and we had SNR $=5 \mathrm{~dB}$. The number of inner iterations 294 was $I_{\text {in }}=2$. As seen in Fig. 3, upon increasing the SNR value, the 295 inner code's EXIT curve shifted upward, and an open tunnel emerged 296 between the inner code and outer code's EXIT curves at SNR = $2975 \mathrm{~dB}$, where the corresponding EXIT trajectory reached the perfect 298 convergence point of $\left(I_{\mathrm{A}}, I_{\mathrm{E}}\right)=(1,1)$ after $I_{\text {out }}=20$ outer iterations. 299 This ensured that an infinitesimally low bit error ratio (BER) was 300 achievable in the simulated SC-based $\mathrm{SM}$ scenario at $\mathrm{SNR}=5 \mathrm{~dB}$.

\section{C. Maximum Achievable Limit}

302 According to the area property of EXIT charts [30], the maximum 303 achievable limit of our FDE-based SC-SM scheme is calculated as 304 follows: $C_{\text {EXIT }}=\mathcal{A}(\rho) R_{\mathrm{SM}-\mathrm{MIMO}}^{\mathrm{(SC}}[\mathrm{bps} / \mathrm{Hz}]$, where $\mathcal{A}(\rho)$ represents the 305 area under the inner code's EXIT curve at $\mathrm{SNR}=\rho$. The benefit of 306 using this metric is that we have the potential of evaluating any SoD 307 detectors in a semianalytical manner, while it is typically a challenging 308 task to derive the theoretical limit of a suboptimal SoD detector. Since 309 the target scenario of our SC-based SM scheme has a long CIR, the 310 theoretical limit of the optimal detector is not attainable due to its 311 excessive calculation complexity.

312 Fig. 4 shows the maximum achievable rates of our SC-based SM 313 scheme, relying on $M=4$ TAs and $N=4$ receive antennas, while 314 the modulation schemes are considered to be quadrature phase-shift 315 keying, 8-PSK, and 16-PSK. The other system parameters are the same 316 as those used in Fig. 3. The associated capacity of the continuous317 input-continuous-output memoryless channel (CCMC) is also shown

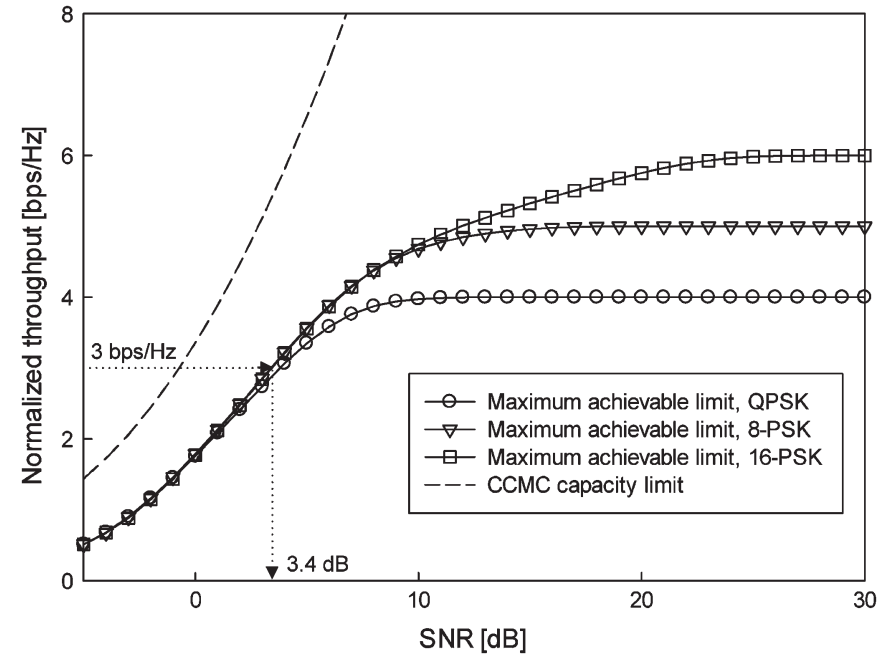

Fig. 4. Maximum achievable limits of our SC-SM-MIMO schemes, employing $M=N=4$ transmit and receive antenna elements and 4-, 8-, and 16-PSK modulation schemes. The associated CCMC capacity limit is also shown.

as a benchmark, representing unconstrained signaling. Observe in 318 Fig. 4 that upon increasing the constellation size from $\mathcal{L}=4$ to $\mathcal{L}=319$ 16 , the maximum achievable limit at high SNR is increased. In each 320 scenario, the curve reached the rate formulated in (3). When employing 321 the half-rate RSC code for the 16-PSK SC-based SM scheme, the 322 limit was reached for an SNR of $\rho=3.4 \mathrm{~dB}$. Since the code's EXIT 323 curve is based on the soft output of the inner code, the conven- 324 tional hard-decision SC-based SM detector is not applicable in this 325 evaluation.

\section{BER PERFORMANCE}

A. Channel-Encoded SC-SM Scheme Aided by Iterative Detection

Here, we investigate the BER of our SC-based SM scheme. The 331 basic system parameters used in our simulations were the same as 332 those in Fig. 3. For simplicity, the estimate of the noise variance 333 $N_{\text {MAP }}$ shown in (4) was set to $N_{0}$. We considered a frequency-selective 334 Rayleigh distributed block-fading channel, where the block length was 335 $K=256$, the CP length was $\nu=32$, and the CIR taps were constant 336 for a block, but were independently faded for the consecutive blocks. ${ }^{5} 337$

Fig. 5 shows the achievable BER of our FDE-aided SC-SM scheme, 338 where the basic system parameters were the same as those used in 339 the EXIT charts in Fig. 3. The number of outer iterations $I_{\text {out }}$ was 340 varied from 0 to 16. Observe in Fig. 5 that upon increasing the number 341 of outer iterations $I_{\text {out }}$, the BER curve significantly improved. In 342 particular, an infinitesimally low BER was achieved for SNR $=5 \mathrm{~dB} 343$ with the aid of $I_{\text {out }}=16$ outer iterations, as predicted by the associated 344 EXIT charts shown in Fig. 3. This is the explicit benefit of the proposed 345 turbo FDE scheme's iterative detection, which would not be attainable 346 by the previous hard-decision SC-SM schemes [18]-[20].

\section{CONCLUSION}

In conclusion, single-RF SM requires SC transmissions, rather 349 than OFDM, for transmission over practical broadband SM-MIMO 350

\footnotetext{
${ }^{5}$ Note that the power penalty per frame imposed by the $\mathrm{CP}$ overhead was as low as $-10 \log _{10}[K /(K+\nu)]=0.51 \mathrm{~dB}$. This can be further reduced by increasing the block length, at the cost of increasing the delay.
} 


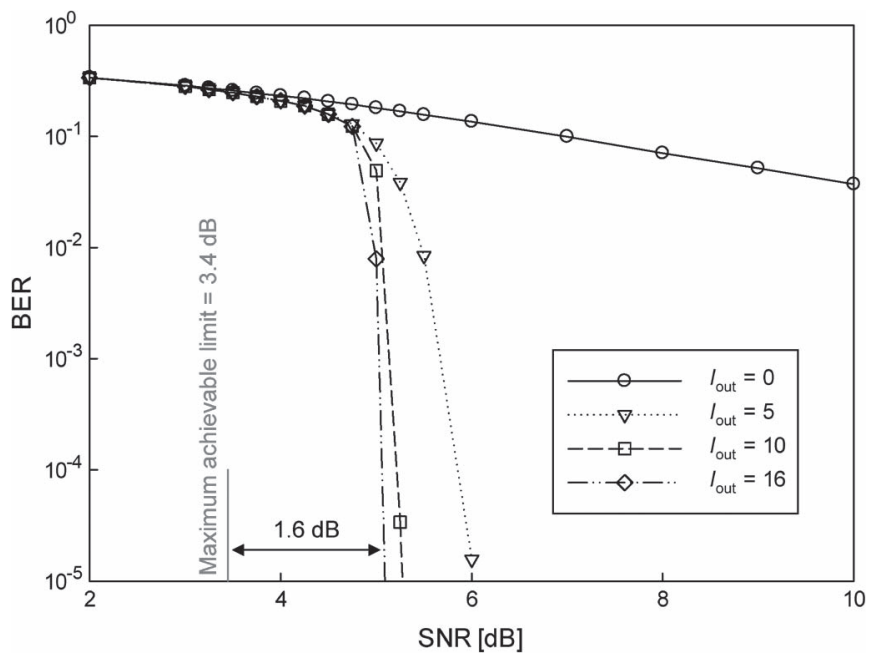

Fig. 5. Achievable BER curves of our FDE-aided SC-SM system, employing $M=N=4$ transmit and receive antenna elements and 16-PSK modulation. The normalized transmission rate of the half-rate channel-encoded system was $R=3 \mathrm{bps} / \mathrm{Hz}$. The interleaver length was $38400 \mathrm{bits}$, we used the half-rate $\operatorname{RSC}(2,1,2)$ code, and the block length was $K=256$.

351 channels. Hence, a novel SoD FDE algorithm was developed for our 352 SM-MIMO scheme. This algorithm enables us to operate in a realistic 353 dispersive fading channel exhibiting a long CIR while attaining a near354 capacity performance. shift keying: A dispersion matrix approach," IEEE Trans. Commun., vol. 58, no. 11, pp. 3219-3230, Nov. 2010.
[10] M. Di Renzo and H. Haas, "A general framework for performance 382 analysis of space shift keying (SSK) modulation for MISO correlated 383 Nakagami-m fading channels," IEEE Trans. Commun., vol. 58, no. 9, 384 pp. 2590-2603, Sep. 2010.

[11] S. Sugiura and L. Hanzo, "Effects of channel estimation on spatial 386 modulation," IEEE Signal Process. Lett., vol. 19, no. 12, pp. 805-808, 387 Dec. 2012.

[12] S. Ganesan, R. Mesleh, H. Haas, C. W. Ahn, and S. Yun, "On the per- 389 formance of spatial modulation OFDM," in Proc. 40th Asilomar Conf. 390 Signals, Syst. Comput., 2006, pp. 1825-1829.

[13] R. Y. Mesleh, H. Haas, S. Sinanovic, C. Ahn, and S. Yun, "Spatial 392 modulation," IEEE Trans. Veh. Technol., vol. 57, no. 4, pp. 2228-2242, 393 Jul. 2008.

[14] M. Kadir, S. Sugiura, J. Zhang, S. Chen, and L. Hanzo, "OFDMA/SC- 395 FDMA aided space-time shift keying for dispersive multiuser scenarios," 396 IEEE Trans. Veh. Technol., vol. 62, no. 1, pp. 408-414, Jan. 2013.

[15] A. Kalis, A. G. Kanatas, and C. B. Papadias, "A novel approach to MIMO 398 transmission using a single RF front end," IEEE J. Sel. Areas Commun., 399 vol. 26, no. 6, pp. 972-980, Aug. 2008.

[16] O. N. Alrabadi, J. Perruisseau-Carrier, and A. Kalis, "MIMO transmission 401 using a single RF source: Theory and antenna design," IEEE Trans. 402 Antennas Propag., vol. 60, no. 2, pp. 654-664, Feb. 2012. virtual MIMO systems," IEEE Signal Process. Lett., vol. 21, no. 4, 405 pp. 390-394, Apr. 2014.

[18] H. A. Ngo, C. Xu, S. Sugiura, and L. Hanzo, "Space-time-frequency 407 shift keying for dispersive channels," IEEE Signal Process. Lett., vol. 18, 408 no. 3, pp. 177-180, Mar. 2011.

[19] P. Som and A. Chockalingam "Spatial modulation and space shift keying 410 in single carrier communication," in Proc. IEEE 23rd Int. Symp. PIMRC, 411 Sep. 2012, pp. 1962-1967.

[20] R. Rajashekar, K. V. S. Hari, and L. Hanzo, "Spatial modulation aided 413 zero-padded single carrier transmission for dispersive channels," IEEE 414 Trans. Commun., vol. 61, no. 6, pp. 2318-2329, Jun. 2013.

[21] F. Pancaldi, G. Vitetta, R. Kalbasi, N. Al-Dhahir, M. Uysal, and 416 H. Mheidat, "Single-carrier frequency domain equalization," IEEE Signal 417 Process. Mag., vol. 25, no. 5, pp. 37-56, Sep. 2008.

[22] K. Ishibashi and S. Sugiura, "Effects of antenna switching on band- 419 limited spatial modulation," IEEE Wireless Commun. Lett., vol. 3, no.4, 420 pp. 345-348, Aug. 2014.

[23] L. Hanzo, T. Liew, B. Yeap, R. Y. S. Tee, and S. X. Ng, Turbo Coding, 422 Turbo Equalisation, and Space-Time Coding for Transmission over Fad- 423 ing Channels. Hoboken, NJ, USA: Wiley, 2011.

[24] S. ten Brink, "Convergence behavior of iteratively decoded parallel con- 425 catenated codes," IEEE Trans. Commun., vol. 49, no. 10, pp. 1727-1737, 426 Oct. 2001.

[25] L. Hanzo, L.-L. Yang, E.-L. Kuan, and K. Yen, Single and Multi-Carrier 428 CDMA: Multi-User Detection, Space-Time Spreading, Synchronisation 429 and Standards, Hoboken, NJ, USA: Wiley, 2003.

430

[26] M. Tüchler, A. Singer, and R. Koetter, "Minimum mean squared error 431 equalization using a priori information," IEEE Trans. Signal Process., 432 vol. 50, no. 3, pp. 673-683, Mar. 2002.

[27] S. Sugiura, S. Chen, and L. Hanzo, "MIMO-aided near-capacity turbo 434 transceivers: Taxonomy and performance versus complexity," IEEE 435 Commun. Surveys Tuts., vol. 14, no. 2, pp. 421-442, May 2012.

[28] L. Hanzo, O. Alamri, M. El-Hajjar, and N. Wu, Near-Capacity Multi- 437 Functional MIMO Systems: Sphere-Packing, Iterative Detection and 438 Cooperation. Hoboken, NJ, USA: Wiley, 2009.

[29] S. ten Brink, "Designing iterative decoding schemes with the extrinsic 440 information transfer chart," AEU Int. J. Electron. Commun., vol. 54, 441 no. 6, pp. 389-398, Nov. 2000.

[30] S. ten Brink, G. Kramer, and A. Ashikhmin, "Design of low-density 443 parity-check codes for modulation and detection," IEEE Trans. Commun., 444 vol. 52, no. 4, pp. 670-678, Apr. 2004. 


\section{AUTHOR QUERIES}

AUTHOR PLEASE ANSWER ALL QUERIES

AQ1 = Please provide keywords.

END OF ALL QUERIES 